\title{
EDITORIAL
}

\section{Assessing the risks of risk sharing}

\author{
Inconclusive initial results from a pioneering risk-sharing scheme in the UK to provide \\ cost-effective access to novel drugs for multiple sclerosis illustrate the challenges of such \\ strategies.
}

In 2002, the National Institute for Health and Clinical Excellence (NICE) in the UK issued guidance on the use of four disease-modifying drugs for multiple sclerosis. The guidance recommended against their use by the National Health Service (NHS), indicating that they were not cost-effective. However, with the aim of providing the drugs - three interferon- $\beta$ agents (Avonex, developed by Biogen-Idec; Betaferon, developed by Bayer-Schering; and Rebif, developed by Merck-Serono) and glatiramer acetate (Copaxone, developed by Teva) more cost-effectively, the UK Department of Health and the manufacturers established a pioneering 'risk-sharing scheme'.

Under the terms of the scheme, the drugs, which had been shown to reduce the risk of disease relapse in placebocontrolled trials lasting up to 2 years, were allowed to be prescribed, conditional on the development of a 10-year monitoring study to collect data on the progression of the disease in treated patients. If any of the individual drugs failed to demonstrate benefits consistent with initial predictions, the subsequent cost of that drug to the NHS would be reduced to restore cost-effectiveness in line with an agreed benchmark.

Now, almost 8 years later, the first interim analysis of the monitoring study has been reported ${ }^{1}$. Disappointingly, with the data so far, it is too early to reach any conclusion about the cost-effectiveness of the drugs studied, raising questions about the limitations of the study. And in view of the results, the Multiple Sclerosis Society - the largest charity for people affected by the disease in the UK - has withdrawn its support for the scheme.

Some of the challenges of the study are readily apparent in the report ${ }^{1}$. From the initiation of recruitment in May 2002 , it took nearly 3 years to recruit the $\sim 5,000-7,000$ patients it was anticipated would be needed. However, only 3,686 of the 5,583 patients registered were eligible for analysis and had any valid data available after their initial assessment, and only 2,901 had valid assessments of disease after 2 years, limiting the strength of statistical analyses of the population. Furthermore, given that a 10-year placebo-controlled study was not deemed appropriate, the economic model for cost-effectiveness was based on a historical dataset from $\sim 1,000$ patients from the 1970s and 1980s that assumes that disability scores only worsen over time, which does not reflect the typical disease course, hampering comparisons with the data for drugs in the scheme.

Given such limitations, it is not surprising that conclusions cannot be drawn about the cost-effectiveness of the four drugs at present. It is hoped that longer, more complete follow-up will be more informative if the data can be collected rigorously. In addition, as well as providing access to new drugs for the patients involved that would otherwise not have been possible, there have already been other benefits of the scheme, including the establishment of a large cohort of patients that could be used for future studies and the development of expertise at specialist treatment centres.

Nevertheless, it is clear overall that multiple sclerosis treatment is not an ideal 'test case' for evaluating innovative risk-sharing schemes to balance limited understanding of the cost-effectiveness of recently approved drugs with the constraints of health-care budgets. In this respect, a recent example ${ }^{2}$ - also from the UK - in which the NHS will only pay for the anticancer drug bortezomib (Velcade, developed by Millennium/Janssen-Cilag) when patients with multiple myeloma show a response to treatment indicated by a biomarker of tumour load, could be more valuable. At least in this case, key aspects such as the ability to obtain the data needed and the linkage between this data and the health gain are much clearer than for drugs for multiple sclerosis.

However, until such information is available, the challenges and costs of implementing and monitoring such schemes are not clear. In addition, their applicability is limited for diseases that lack suitable biomarkers, or for which the data needed for comparison are limited, as with multiple sclerosis. So, if future access to new drugs in general is not to be increasingly constrained by a rising 'fourth hurdle' of cost-effectiveness, other approaches, such as aiming to achieve closer alignment of the requirements of regulators and payers in both preand post-market clinical development, deserve further consideration.

\footnotetext{
1. Boggild, M. et al. Multiple sclerosis risk sharing scheme: two year results of clinical cohort study with historical comparator. BMJ 339, b4677 (2009).

2. Hughes, B. Novel risk-sharing scheme puts the spotlight on biomarkers. Nature Rev. Drug Discov. 6, 945 (2007).
} 